\title{
Heat Kernel Expansion for Semitransparent Boundaries
}

\author{
M. Bordag! and D.V. Vassilevich? \\ Institute for Theoretical Physics, Leipzig University \\ Augustusplatz 10/11, 04109 Leipzig, Germany
}

\begin{abstract}
We study the heat kernel for an operator of Laplace type with a $\delta$ function potential concentrated on a closed surface. We derive the general form of the small $t$ asymptotics and calculate explicitly several first heat kernel coefficients.
\end{abstract}

\section{Introduction}

Singular potentials are a frequently used idealization of physical situations allowing for an easier (and, sometimes, explicit) solution while keeping the essential features of the problem. The best studied cases of singular potentials are the delta function potentials concentrated at isolated points, which describe contact interactions of particles (for a review, see [1]). Rigorous analysis of such potentials was initiated by the paper by Berezin and Faddeev [2] and has developed later in a mature mathematical discipline [1]. Other cases of singular potentials include also cosmic strings and other topological defects [3] and problems related to the black hole entropy [4]. We like also to mention a recent work on the boundary discontinuities [5].

With respect to the Casimir effect, a delta function shaped potential provides the simplest generalization of the conductor boundary conditions towards inclusion of more realistic properties of the walls like partial transparency. For a scalar and a spinor field with plane boundaries this problem has been investigated in [6], for moving partly transmitting mirrors in [7]. An interesting approach using 'semihard' and 'weak' boundaries is developed in [8]. In all those cases it is crucial to know the ultra violet divergences in order to find out the structure of necessary counterterms. This is equivalent to the investigation of the corresponding heat kernel asymptotics. As it is known [9], this is an expansion with respect to integer powers of the proper time parameter $t$ (see below) for Laplace type operators on closed manifolds and to half integer powers, i.e., to powers of $\sqrt{t}$, on manifolds with boundaries and Dirichlet or Robin boundary conditions. For more complicated pseudo differential operators powers of $\ln t$ may appear.

\footnotetext{
${ }^{1}$ e-mail: Michael.Bordag@itp.uni-leipzig.de

${ }^{2}$ On leave from Department of Theoretical Physics, St.Petersburg University, 198904 St.Petersburg, Russia. e-mail: Dmitri.Vassilevich@itp.uni-leipzig.de
} 
The present paper is devoted to singularities which are located on closed hypersurfaces of dimension $m-1$ where $m$ is dimension of the underlying manifold. Apart from the quantum mechanical problem of a particle in the space with semitransparent boundaries (for a recent calculation of the vacuum energy for such system see e.g. [10) possible physical application also include fermions on a background of a magnetic tube [11] and photons interacting with dielectric bodies [12, 13]. In all these cases the dynamics is described by a second order differential operator of Laplace type supplemented by certain matching conditions on a surface. Our primary interest is in the heat kernel asymptotics. They govern the short time asymptotics of quantum mechanical propagators, the ultra violet divergences and the large mass expansion in quantum field theory.

The heat kernel coefficients for singular potentials cannot be obtained in general as limiting cases of smooth configurations. This is clear already from the fact that the heat kernel expansion for a smooth potential contains powers of the potential taken at the same point. Such expressions become ill-defined in the delta-function limit. In our previous paper 13n we have observed a surprising property that sometimes the ultra violet behavior of a system with $\delta$-function potential is less singular than that of corresponding "smooth" system.

Let us proceed with basic definitions. Let $M$ be a smooth Riemannian manifold of dimension $m$. Let $\Sigma$ be a smooth closed submanifold of co-dimension 1 . Let $\mathcal{V}$ be a vector bundle over $M$. Let $E$ and $V$ be endomorphisms of $\mathcal{V}$ and $\left.\mathcal{V}\right|_{\Sigma}$ respectively. In more "physical" notations, $E$ and $V$ are matrix valued functions bearing spin and internal indices. In the present paper we study the heat kernel expansion for the operator

$$
D=-\left(\nabla^{2}+E(x)+\delta_{\Sigma} V(x)\right)=D_{0}-\delta_{\Sigma} V(x) .
$$

Let $d x$ and $d y$ be the Riemannian volume elements on $M$ and $\Sigma$. We normalize the function $\delta_{\Sigma}$ in such a way that for any smooth function $f$

$$
\int_{M} \delta_{\Sigma} f(x) d x=\int_{\Sigma} f(y) d y
$$

We adopt the following short hand notations for the integrals

$$
\int_{M} d x F(x)=\{F\}[M], \quad \int_{\Sigma} d y F(y)=\{F\}[\Sigma] .
$$

We can choose the coordinates in such a way that in the vicinity of $\Sigma$ the metric has the form

$$
g_{i j} d x^{i} d x^{j}=\left(d x^{m}\right)^{2}+g_{a b} d x^{a} d x^{b} .
$$

The second fundamental form of $\Sigma$ is $L_{a b}=\frac{1}{2} \partial_{m} g_{a b}$. We suppose that $x^{m}=0$ on $\Sigma$. Our notations are the same as in Ref. [14]. $R_{i j k l}$ are the components of the 
Riemann curvature tensor. With our sign conventions, $R_{1212}$ is negative on the standard sphere in Euclidean space. The Ricci tensor $\rho$ and the scalar curvature $\tau$ are given by

$$
\rho_{i j}:=R_{i k k j} \quad \tau=\rho_{i i}=R_{i k k i} .
$$

Let $\rho^{2}:=\rho_{i j} \rho_{i j}$ and $R^{2}:=R_{i j k l} R_{i j k l}$ be the norm of the Ricci and full curvature tensors. Let $\Omega_{i j}$ be the endomorphism valued components of the curvature of the connection on $\mathcal{V}$. In physical language, $\Omega_{i j}$ is the field strength for the Yang-Mills and spin-connections. Let ';' denote multiple covariant differentiation with respect to the Levi-Civita connection of $M$ and let ' $'$ ' denote multiple tangential covariant differentiation on the boundary with respect to the Levi-Civita connection of $\Sigma$; the difference between these two is measured by the second fundamental form. For example, $f_{; j j}=f_{; m m}+f_{; a a}=f_{; m m}+f_{: a a}-L_{a a} f_{; m}$.

A mathematically rigorous way to define the spectral problem for the operator $D$ is to replace it by the spectral problem for $D_{0}$ for $x \notin \Sigma$ supplemented by the conditions on $\Sigma$ (see e.g. [1])

$$
\begin{aligned}
& \phi(-0)=\phi(+0) \\
& \nabla_{m} \phi(-0)-\nabla_{m} \phi(+0)=V \phi(0)
\end{aligned}
$$

with the short hand notation $\phi( \pm 0)=\lim _{x^{m} \rightarrow \pm 0} \phi(x)$.

The heat kernel $K(x, y ; t)$ is a solution of the heat equation

$$
\left(\partial_{t}+D_{0}\right) K(x, y ; t)=0
$$

with the initial condition

$$
K(x, y ; 0)=\delta(x, y),
$$

which satisfies the matching conditions on $\Sigma$ following from (6) and (7).

We are interested in the integrated heat kernel

$$
K(f, D ; t)=\{f(x) K(x, x ; t)\}[M]=\operatorname{Tr}(f \exp (-t D)) .
$$

On manifolds with boundary with local boundary conditions there is an asymptotic expansion as $t \rightarrow+0$

$$
K(f, D ; t)=\sum_{n=0} a_{n}(f, D) t^{(n-m) / 2},
$$

where the coefficients $a_{n}(f, D)$ are volume and surface integrals of local invariants. The existence of the asymptotic expansion (11) is usually considered as granted. There are however important exceptions (besides "genuine" pseudo differential operators, the square root of Laplacian for instance), such as the boundary value problem for spectral boundary conditions and $\delta$-function potentials with pointlike support on manifolds with dimension $m \geq 2$. In such cases $\ln t$ terms can appear in the asymptotic expansion [15, 16, 17]. To the best of our knowledge, 
the existence of the expansion (11) for the problem considered here has never been stated before.

This paper is organized as follows. In the next section we derive an integral equation for the heat kernel and show validity of the asymptotic expansion (11). In section 3 we calculate the heat kernel asymptotics for the particular case when $\Sigma$ is a sphere in $\mathrm{R}^{m}$. In section 5 we derive explicit expressions for the heat kernel coefficients $a_{n}(f, D), n \leq 5$ for the most general form of the operator $D$. To this end we use the particular case calculations of section 4 and functorial properties of the heat kernel.

\section{General structure of the heat kernel}

To study the general structure of the heat kernel expansion we use an integral equation similar to that proposed by Gaveau and Schulman [18 for the onedimensional $\delta$-potential:

$$
K(x, y ; t)=K_{0}(x, y ; t)+\int_{0}^{t} d s \int_{\Sigma} d z K_{0}(x, z ; t-s) V(z) K(z, y ; s)
$$

where $K_{0}(x, y ; t)$ denotes the heat kernel corresponding to the operator $D_{0}$ with $V=0$.

The equation (12) has a solution in the form of the power series in $V$ :

$$
\begin{array}{r}
K(x, y ; t)=K_{0}(x, y ; t)+\sum_{n=1}^{\infty} \int_{0}^{t} d s_{n} \int_{0}^{s_{n}} d s_{n-1} \ldots \int_{0}^{s_{2}} d s_{1} \int_{\Sigma} d z_{n} \ldots \int_{\Sigma} d z_{1} \\
\times K_{0}\left(x, z_{n} ; t-s_{n}\right) V\left(z_{n}\right) K_{0}\left(z_{n}, z_{n-1} ; s_{n}-s_{n-1}\right) \ldots V\left(z_{1}\right) K_{0}\left(z_{1}, y ; s_{1}\right) .
\end{array}
$$

The equation (12) can be obtained formally as a limiting case of the smooth potential. Instead of investigating this limiting procedure we prefer to check directly that the heat kernel defined by (12) satisfies the heat equation (8) with the initial condition (9) and boundary conditions which follow from (6) and (7). The

initial condition (9) is evident from the eq. (13). Only the first term contributes at $t=0$ if $x, y \notin \Sigma$. The heat equation (8) can be checked by a direct calculation. The first of the matching conditions (6) just expresses the fact that the heat kernel $K_{0}(x, y ; t)$ is smooth enough. The second condition (7) is a bit less trivial. Let $y \notin \Sigma$. Then the following sequence of transformations holds:

$$
\begin{aligned}
& \nabla_{m}^{x} K(-0, y ; t)-\nabla_{m}^{x} K(+0, y ; t)= \\
&=-\lim _{\epsilon \rightarrow 0} \int_{-\epsilon}^{\epsilon} d x^{m} \nabla_{m}^{2} \int_{0}^{t} d s \int_{\Sigma} d z K_{0}(x, z ; t-s) V(z) K(z, y ; s) \\
& \quad=\lim _{\epsilon \rightarrow 0} \int_{-\epsilon}^{\epsilon} d x^{m} D_{0}^{x} \int_{0}^{t} d s \int_{\Sigma} d z K_{0}(x, z ; t-s) V(z) K(z, y ; s)
\end{aligned}
$$




$$
\begin{aligned}
& =\lim _{\epsilon \rightarrow 0} \int_{-\epsilon}^{\epsilon} d x^{m} \int_{0}^{t} d s \int_{\Sigma} d z\left(\partial_{s} K_{0}(x, z ; t-s)\right) V(z) K(z, y ; s) \\
& =\lim _{\epsilon \rightarrow 0} \int_{-\epsilon}^{\epsilon} d x^{m} \int_{0}^{t} d s \int_{\Sigma} d z K_{0}(x, z ; t-s) V(z) D_{0}^{y} K(z, y ; s) \\
& +\lim _{\epsilon \rightarrow 0} \int_{-\epsilon}^{\epsilon} d x^{m} \int_{\Sigma} d z\left[\delta(x, z) V(x) K(z, y ; t)-K_{0}(x, z ; t) V(z) \delta(z, y)\right] \\
& =V\left(x^{a}, 0\right) K\left(\left(x^{a}, 0\right), y ; t\right)
\end{aligned}
$$

The main advantage of the representation (13) is that the small $t$ behavior of $K(x, y ; t)$ is defined through the small $t$ behavior of $K_{0}(x, y ; t)$ which is known in some detail. To simplify the notations we do not write down explicitly the volume elements here and the parallel transport matrices later on. We also drop all matrix indices.

We are interested in the integrated heat kernel $K(t)=\int d x K(x, x ; t)$, where we put the smearing function $f=1$ for simplicity. The integration over $x$ can be performed by using the equation

$$
\int_{M} d x K_{0}\left(x, y_{1} ; \tau_{1}\right) K_{0}\left(y_{2}, x ; \tau_{2}\right)=K_{0}\left(y_{1}, y_{2} ; \tau_{1}+\tau_{2}\right)
$$

which follows from the evident operator identity $e^{-\tau_{1} D_{0}} e^{-\tau_{2} D_{0}}=e^{-\left(\tau_{1}+\tau_{2}\right) D_{0}}$. We have

$$
\begin{array}{r}
K(t)=K_{0}(t)+\sum_{n=1}^{\infty} \int_{0}^{t} d s_{n} \int_{0}^{s_{n}} d s_{n-1} \ldots \int_{0}^{s_{2}} d s_{1} \int_{\Sigma} d z_{n} \ldots \int_{\Sigma} d z_{1} \\
\times K_{0}\left(z_{1}, z_{n} ; t+s_{1}-s_{n}\right) V\left(z_{n}\right) K_{0}\left(z_{n}, z_{n-1} ; s_{n}-s_{n-1}\right) \ldots V\left(z_{1}\right) .
\end{array}
$$

It is instructive to calculate explicitly several first terms of the expansion (15). In the linear order in $V$ one immediately gets

$$
K_{1}(t)=t \int_{\Sigma} d z K_{0}(z, z ; t) V(z)
$$

This equation means that the liner order in $V$ can be obtained from the asymptotic expansion for the heat kernel in which a smooth potential is replaced by the singular one. Such simple relation does not hold at higher orders in $V$.

In analyzing the order $V^{2}$ contributions we suppose that $D_{0}$ is just the standard scalar Laplacian in $\mathrm{R}^{m}$, neglect derivatives of $V$ and suppose that $\Sigma$ is flat. However, we turn to a bit more general case allowing $\Sigma$ to be of dimension $m-k$. In this particular case $K_{0}(x, y ; t)=(4 \pi t)^{-m / 2} \exp \left(-(x-y)^{2} / 4 t\right)$ and

$$
K_{2}(t)=(4 \pi)^{-\frac{m+k}{2}} t^{\frac{m-k}{2}} \int_{\Sigma} d z V(z)^{2} \int_{0}^{t} d s_{2} \int_{0}^{s_{2}} d s_{1}\left(\left(t-s_{2}+s_{1}\right)\left(s_{2}+s_{1}\right)\right)^{-\frac{k}{2}}
$$


For $k>1$ the integrals over $s$ are divergent. This shows that the proposed method can not be extended in particular to $\delta(r)$ potentials in $\mathbf{R}^{m}$ with $m \geq 2$ for which the expansion (11) is known to break down [16, 17]). Return to the subject of our study, $k=1$. All integrals are easily calculated giving

$$
K_{2}(t)=\frac{t}{(4 \pi t)^{(m-1) / 2}} \frac{1}{8} \int_{\Sigma} d z V(z)^{2}+\ldots
$$

where we have omitted all higher order terms.

To calculate the other terms in the expansion (15) one can use the following strategy. Let us substitute the small $s$ asymptotic expansion for the $K_{0}(x, y ; s)$

$$
K_{0}(x, y ; s) \sim \frac{\exp (-\sigma(x, y) / 2 s)}{(4 \pi s)^{m / a}}\left(A_{0}^{(0)}(x, y)+s A_{2}^{(0)}(x, y)+\ldots\right),
$$

where $\sigma(x, y)$ is the half square of the geodesic distance between $x$ and $y, A_{2 i}^{(0)}(x, y)$ are the heat kernel coefficients for the operator $D_{0}$. Contributions of largely separated points to (15) are exponentially damped. Therefore, we may expand $A_{2 i}^{(0)}\left(z_{j}, z_{j-1}\right)$ and $\sigma\left(z_{j}, z_{j-1}\right)$ in Taylor series in $\left(z_{j}-z_{j-1}\right)$. The potentials $V\left(z_{j}\right)$ are to be expanded around certain point, say $z_{1}$. At the end, the expression under the integrals in (15) will become a sum of the monomials

$$
\begin{aligned}
& \exp \left(-\frac{\left(z_{1}-z_{n}\right)^{2}}{4\left(t-s_{1}+s_{n}\right)}-\ldots-\frac{\left(z_{2}-z_{1}\right)^{2}}{4\left(s_{2}-s_{1}\right)}\right) I^{(N)}\left(V, \ldots ; z_{1}\right) \\
& \quad \times \frac{\left(z_{1}-z_{n}\right)^{N_{1}}}{\left(t-s_{1}+s_{n}\right)^{M_{1}}} \cdots \frac{\left(z_{2}-z_{1}\right)^{N_{n}}}{\left(s_{2}-s_{1}\right)^{M_{n}}}
\end{aligned}
$$

where $I^{(N)}$ is a local invariant functional of $V$, geometric invariants and their derivatives calculated at the point $z_{1}$. Negative powers of $\left(s_{j}-s_{j-1}\right)$ appear due to the expansion of the $\sigma\left(z_{j}-z_{j-1}\right)$ in the exponentials. It is easy to see that $M_{j} \leq N_{j} / 2$.

Integrals over $z_{i}$, except for the last one over $z_{1}$, can be calculated with the help of the relation

$$
\int_{\mathbf{R}^{n}} d x \exp \left(-\frac{\left(y_{1}-x\right)^{2}}{4 \alpha}-\frac{\left(x-y_{2}\right)^{2}}{4 \beta}\right)=\left(\frac{4 \pi \alpha \beta}{\alpha+\beta}\right)^{\frac{n}{2}} \exp \left(-\frac{\left(y_{1}-y_{2}\right)^{2}}{4(\alpha+\beta)}\right)
$$

with positive real parameters $\alpha$ and $\beta$. Note that eq. (21) is just a particular case of (14) when $D_{0}$ is the flat space Laplacian. Integrals of even powers of $\left(x-y_{1}\right)$ with the same exponential weight are obtained by differentiation of (21) with respect to $\alpha$. Odd powers of $x$ can be integrated by using the following obvious relation

$$
\int_{\mathbf{R}^{n}} d x\left(x^{a}-\frac{\beta y_{1}^{a}+\alpha y_{2}^{a}}{\alpha+\beta}\right) \exp \left(-\frac{\left(y_{1}-x\right)^{2}}{4 \alpha}-\frac{\left(x-y_{2}\right)^{2}}{4 \beta}\right)=0 .
$$


Before integrating over $s_{j}$ let us introduce rescaled variables $\tilde{s}_{j}=s_{j} / t$. This enables us to extract an overall power of the proper time $t$. Integrals over $\tilde{s}_{j}$ will give just numerical factors. One can easily see that the strongest possible singularity of the integrand has the form $\left(\left(1-\tilde{s}_{n}+\tilde{s}_{1}\right) \ldots\left(\tilde{s}_{2}-\tilde{s}_{1}\right)\right)^{-\frac{1}{2}}$. This singularity is integrable. Hence the integral over $\tilde{s}_{j}$ always exists. After all the integrations have been done one obtains $\int_{\Sigma} d z_{1} I^{(N)}\left(V, \ldots ; z_{1}\right)$ multiplied by a numerical coefficient and a power of $\sqrt{t}$.

Generalization of the procedure proposed in this section for the case of nonunit smearing function $f$ is obvious. Therefore, we have demonstrated that for the spectral problem considered in this paper the asymptotic expansion (11) is valid where the coefficients $a_{n}(f, D)$ are integrals over $M$ and $\Sigma$ of local invariants. Volume terms are the same as in the heat kernel expansion for the operator $D_{0}$.

\section{$3 \quad$ Penetrable spherical shell}

Before calculating the heat kernel expansion for generic form of the operator $D$ consider a particular case of the constant potential $V$ with the support on a spherical shell of the radius $R$ :

$$
V(x) \delta_{\Sigma}=-\frac{\alpha}{R} \delta(r-R) .
$$

Here $r$ is the radial coordinate. Let $D_{0}$ in (1D) be the standard scalar Laplacian in $\mathrm{R}^{m}$. After separating the angular variables the eigenvalue equation takes the form

$$
\left(-\frac{\partial^{2}}{\partial r^{2}}-\frac{(m-1)}{r} \frac{\partial}{\partial r}+\frac{l(l+m-2)}{r^{2}}\right) \phi=k^{2} \phi,
$$

where $l=0,1,2, \ldots$ is the orbital momentum. After the substitution $\phi(r)=$ $r^{(2-m) / 2} \psi(r)$ the equation takes the form

$$
\left(-\frac{\partial^{2}}{\partial r^{2}}-\frac{1}{r} \frac{\partial}{\partial r}+\frac{\nu^{2}}{r^{2}}\right) \psi=k^{2} \psi
$$

where the notation $\nu=l+\frac{m-2}{2}$ is introduced.

Using the standard techniques [19], the zeta function associated with this operator can be written in the form

$$
\zeta(s)=\int_{0}^{\infty} \frac{\mathrm{d} t}{t} \frac{t^{s}}{\Gamma(s)} K(t),
$$

where $K(t)$ is the integrated heat kernel. The function $\Gamma(s) \zeta(s)$ has simple poles in $s=\frac{d}{2}-N\left(N=0, \frac{1}{2}, 2, \ldots\right)$ whose residua are determined by the expansion of the heat kernel for $t \rightarrow 0$. It reads

$$
K(t) \sim \sum_{n \geq 0} a_{n} t^{(n-m) / 2} .
$$


It can be easily seen that the coefficients are related to the residua by means of

$$
a_{n}=\underset{s=\frac{m-n}{2}}{\operatorname{Res}} \Gamma(s) \zeta(s) .
$$

These heat kernel coefficients can be obtained by calculating the zeta function starting from the differential equation, Eq. (25). The zeta function of the operator $D$ can be expressed in the form

$$
\zeta(s)=\frac{\sin \pi s}{\pi} \sum_{l=0}^{\infty} D_{l} \int_{0}^{\infty} \mathrm{d} k k^{-2 s} \frac{\partial}{\partial k} \ln f_{l}(i k),
$$

where $f_{l}(k)$ is the Jost function of the scattering problem corresponding to the operator $D$ and

$$
D_{l}=\frac{(2 l+m-2)(l+m-3) !}{l !(m-2) !} \quad m \geq 3
$$

is the multiplicity of the orbital eigenvalues. This representation, as it stands, is valid for $\Re s>\frac{m}{2}$. The Jost function reads [13]

$$
f_{l}(i k)=1+\alpha I_{\nu}(k) K_{\nu}(k) .
$$

In Eq. (29) the contribution resulting from the empty space (it is independent from $\alpha$ ) is dropped. By this reason there is no contribution corresponding to the coefficient $a_{0}$ there.

In fact, we need the residua of the function $\Gamma(s) \zeta(s)$. These are delivered when inserting the uniform asymptotic expansion for $k \rightarrow \infty$ and $l \rightarrow \infty$ of the Jost function into Eq. (29). The latter can be obtained simply by inserting the known expansions of the Bessel functions into (31). This expansion can be written in the form

$$
\ln f_{l}(i k)=\sum_{n, i} X_{n, i} t^{i} \nu^{-n} .
$$

Here, $n=1, \ldots, N_{m}$, and $N_{m}$ is the number of the highest heat kernel coefficient requested to be calculated. The coefficients $X_{n . i}$ are numbers, some first given in the appendix. The notation $t=\sqrt{1+(k / \nu)^{2}}$ is used. When inserting (32) into the r.h.s. of Eq. (29), the change of variables $k \rightarrow \nu k$ can be made after what the expression factorizes. The integral can be calculated easily. It reads

$$
\int_{0}^{\infty} \mathrm{d} k k^{-2 s} \frac{\partial}{\partial k} t^{i}=-\frac{\Gamma(1-s) \Gamma(s+i / 2)}{\Gamma(i / 2)} .
$$

The sum over $l$ takes the form

$$
\zeta(d, n)=\sum_{l=0}^{\infty} D_{l} \nu^{-2 s-n} .
$$


The functions $\zeta(d, n)$ can be expressed in terms of Riemann and Hurwitz zeta functions. They are shown in the appendix.

Now the heat kernel coefficients can be expressed in the form

$$
a_{N}=-\operatorname{Res}_{s=\frac{d-N}{2}} \sum_{n=1}^{N_{m}} \zeta(d, n) \sum_{i} X_{n, i} \frac{\Gamma(s+i / 2)}{\Gamma(i / 2)} \quad\left(N=1, \frac{3}{2}, 2, \frac{5}{2}, \ldots, N_{m}\right) .
$$

The sum over $i$ runs from $i=n$ to $i=n+4\left(\left[\frac{n+1}{2}\right]-1\right)$ where [...] denotes the integer part. In this form they can be calculated immediately using one of the standard computer systems for analytical calculations. As a results, we obtain that $a_{1}=0$ in any dimension. Other coefficients read for $m=3$ :

$$
\begin{aligned}
& a_{2}=\frac{-\alpha}{2 \sqrt{\pi}} \quad a_{3}=\frac{\alpha^{2}}{8} \\
& a_{4}=\frac{-\alpha^{3}}{12 \sqrt{\pi}} \quad a_{5}=\frac{\alpha^{4}}{64} \\
& a_{3}=\frac{-\left(\alpha^{3}\left(4+21 \alpha^{2}\right)\right)}{2520 \sqrt{\pi}} \quad a_{7}=\frac{\alpha^{4}\left(1+2 \alpha^{2}\right)}{1536},
\end{aligned}
$$

for $m=4$ :

$$
\begin{aligned}
& a_{2}=\frac{-\alpha}{8} \quad a_{3}=\frac{\alpha^{2} \sqrt{\pi}}{32} \\
& a_{4}=\frac{-\alpha^{3}}{48} \quad a_{5}=\frac{-\left(\alpha^{2}\left(3-4 \alpha^{2}\right) \sqrt{\pi}\right)}{1024} \\
& a_{6}=\frac{-\left(\alpha^{3}\left(-8+7 \alpha^{2}\right)\right)}{3360} \quad a_{7}=\frac{-\left(\alpha^{2}\left(135+168 \alpha^{2}-128 \alpha^{4}\right) \sqrt{\pi}\right)}{393216},
\end{aligned}
$$

for $m=5$ :

$$
\begin{aligned}
& a_{2}=\frac{-\alpha}{12 \sqrt{\pi}} \quad a_{3}=\frac{\alpha^{2}}{48} \\
& a_{4}=\frac{-\alpha^{3}}{72 \sqrt{\pi}} \quad a_{5}=\frac{\alpha^{2}\left(-2+\alpha^{2}\right)}{384} \\
& a_{6}=\frac{-\left(\alpha^{3}\left(-24+7 \alpha^{2}\right)\right)}{5040 \sqrt{\pi}} \quad a_{7}=\frac{-9 \alpha^{4}+2 \alpha^{6}}{9216},
\end{aligned}
$$

for $m=6$ :

$$
\begin{aligned}
& a_{2}=\frac{-\alpha}{64} \quad a_{3}=\frac{\alpha^{2} \sqrt{\pi}}{256} \\
& a_{4}=-\frac{\alpha^{3}}{384} \quad a_{5}=-\frac{\alpha^{2}\left(15-4 \alpha^{2}\right) \sqrt{\pi}}{8192} \\
& a_{6}=\frac{-\left(\alpha^{3}\left(-20+3 \alpha^{2}\right)\right)}{11520} \quad a_{7}=\frac{\alpha^{2}\left(945-1160 \alpha^{2}+128 \alpha^{4}\right) \sqrt{\pi}}{3145728}
\end{aligned}
$$


and for $m=7$ :

$$
\begin{aligned}
& a_{2}=\frac{-\alpha}{120 \sqrt{\pi}} \quad a_{3}=\frac{\alpha^{2}}{480} \\
& a_{4}=\frac{-\alpha^{3}}{720 \sqrt{\pi}} \quad a_{5}=\frac{\alpha^{2}\left(-6+\alpha^{2}\right)}{3840} \\
& a_{6}=\frac{-\left(\alpha^{3}\left(-76+7 \alpha^{2}\right)\right)}{50400 \sqrt{\pi}} \quad a_{7}=\frac{\alpha^{2}\left(27-15 \alpha^{2}+\alpha^{4}\right)}{46080} .
\end{aligned}
$$

\section{Calculation of the heat kernel asymptotics}

The information on the general structure of the heat kernel asymptotic obtained in Sec. 2 can be summarized in the following Lemma.

Lemma 1. 1) Let $N^{\nu}(f)=f_{; m . . . m}$ be the $\nu^{\text {th }}$ normal covariant derivative. There exist invariant local formulae $a_{n, \nu}(y, D)$ so that:

$$
a_{n}(f, D)=\left\{a_{n}\left(f, D_{0} ; x\right)\right\}[M]+\left\{\sum_{0 \leq \nu \leq n-1} N^{\nu}(f) a_{n, \nu}(y, D)\right\}[\Sigma]
$$

2) If we expand $a_{n, \nu}$ with respect to a Weyl basis, the coefficients depend on the dimension $m$ only through a normalizing constant.

3) Consider a transformation which changes the sign before the $m$-th components of all vector and tensor fields and reverses the sign of the extrinsic curvature $L_{a b}$. Under this transformation $a_{n, \nu} \rightarrow(-1)^{\nu} a_{n, \nu}$.

4) In the linear order of $V$

$$
a_{n}(f, D)=\left\{V(z) \frac{\delta}{\delta E(z)} a_{n}\left(f, D_{0}\right)\right\}[\Sigma] .
$$

Proof. First assertion is now evident. Volume terms $\left\{a_{n}\left(f, D_{0} ; x\right)\right\}[M]$ are given in the Appendix B. One can also observe that coefficients before monomials constructed from the geometric invariants depend on the dimension $m$ only through the factor $(4 \pi)^{-m / 2}$. A more simple way to prove the second assertion is to consider product spaces $M=S^{1} \times M_{1}$ and $\Sigma=S^{1} \times \Sigma^{1}$ exactly repeating the corresponding proof for manifolds with boundaries [9]. Assertion 3) follows from the fact that we can repeat all calculation of section 2 with the replacement $x^{m} \rightarrow-x^{m}$. The last statement of Lemma 1 is just a trivial generalization of the equation (16) for non-unit smearing function $f$. Indeed, it is sufficient to represent the variation on the r.h.s. of (42) as

$$
\frac{\delta}{\delta E(z)} K_{0}(t)=\int_{\mathcal{M}} d x \int_{0}^{t} d s f(x) K_{0}(z, x ; t-s) K_{0}(x, z ; s) .
$$

Assertion 4) is now evident. 
Now we can determine several first heat kernel coefficient up to a few yet undetermined constants.

Lemma 2. There exist universal constants $c_{3,1}, \ldots, c_{5,10}$ such that

$$
\begin{aligned}
a_{0}(f, D)= & a_{0}\left(f, D_{0}\right) \\
a_{1}(f, D)= & 0 \\
a_{2}(f, D)= & a_{2}\left(f, D_{0}\right)+(4 \pi)^{-m / 2}\{f V\}[\Sigma] \\
a_{3}(f, D)= & (4 \pi)^{-(m-1) / 2}\left\{c_{3,1} f V^{2}\right\}[\Sigma] \\
a_{4}(f, D)= & a_{4}\left(f, D_{0}\right)+(4 \pi)^{-m / 2}\left\{c_{4,1} f V^{3}+\frac{1}{6} f \tau V+f E V\right. \\
& \left.+\frac{1}{6} f V_{: a a}-\frac{1}{6} f_{; m} V L_{a a}+\frac{1}{6} f_{; m m} V\right\}[\Sigma] \\
a_{5}(f, D)= & (4 \pi)^{-(m-1) / 2}\left\{c_{5,1} f V^{4}+c_{5,2} f \tau V^{2}+c_{5,3} \rho_{m m} V^{2}\right. \\
& +c_{5,4} f V^{2} E+c_{5,5} f V^{2} L_{a a} L_{b b}+c_{5,6} f V^{2} L_{a b} L_{a b}+c_{5,7} f V_{: a a} V \\
& \left.+c_{5,8} f V_{: a} V_{: a}+c_{5,9} f_{; m} V^{2} L_{a a}+c_{5,10} f_{; m m} V^{2}\right\}[\Sigma]
\end{aligned}
$$

Proof. According to Lemma 1.1) and 2) any coefficient $a_{n}$ contains all local invariants of appropriate dimension. Some of the invariants, like e.g. $f V L_{a a}$, $f_{; m} V$ etc are ruled out by Lemma 1.3). All terms linear in $V$ are determined by Lemma 1.4).

Lemma 3. $c_{3,1}=\frac{1}{8}, c_{4,1}=\frac{1}{6}, c_{5,1}=\frac{1}{64}, c_{5,4}=\frac{1}{8}, c_{5,5}=-\frac{1}{256}, c_{5,6}=\frac{1}{128}$.

Proof. The coefficients $c_{3,1}, c_{4,1}, c_{5,1}, c_{5,5}$ and $c_{5,6}$ are easily calculated using the example of the delta-potential on the sphere of the previous section. To calculate the coefficient $c_{5,4}$ consider the case then $E=e \mathbf{1}$ is a constant proportional to the unit matrix. In this case $K(f ; t)=\left.K(f ; t)\right|_{E=0} \exp (t e)$. This immediately gives $c_{5,4}=c_{3,1}=\frac{1}{8}$.

Several more universal constants can be calculated by a reduction to Dirichlet and Neumann boundary value problems. All necessary definitions and explicit expressions for the heat kernel coefficient for that problems can be found in the Appendix B.

Lemma 4. 1) Let $M=\Sigma \times[-a, a]$. Let $\nabla_{m}=\partial_{m}$ and let all geometric invariants and the smearing function $f$ be symmetric under $x^{m} \rightarrow-x^{m}$. We suppose that $f$ and a sufficient number of its derivatives vanish at $x^{m}= \pm a$. Then $a_{n}(f, D)=a_{n}\left(f, D_{0}, \mathcal{B}^{-}\right)+a_{n}\left(f, D_{0}, \mathcal{B}^{+}\right)$where the heat kernel coefficients on the right hand side are calculated on $\Sigma \times[0, a]$, and $S=\frac{1}{2} V$.

Proof. Since reflection of the $m$ th coordinate commutes with $D$ one can subdivide the spectral resolution in the two sets $\left(\lambda_{ \pm}^{N}, \phi_{ \pm}^{N}\right)$ with normalized eigenfunctions $\phi_{ \pm}^{N}\left(-x^{m}\right)= \pm \phi_{ \pm}^{N}\left(x^{m}\right)$. Then the heat kernel becomes

$$
K(f ; t)=K_{-}(f ; t)+K_{+}(f ; t)
$$

where

$$
K_{ \pm}(f ; t)=\int_{M} d x f(x) \sum_{N} \exp \left(-t \lambda_{ \pm}\right) \phi_{ \pm}(x)^{2}
$$




$$
=\int_{0}^{a} d x^{m} \int_{\Sigma} d z f\left(x^{m}, z\right) \sum_{N} \exp \left(-t \lambda_{ \pm}\right)\left(\sqrt{2} \phi_{ \pm}\left(x^{m}, z\right)\right)^{2} .
$$

Now we observe that $\sqrt{2} \phi_{ \pm}$are normalized eigenfunctions of the operator $D_{0}$ on $\Sigma \times[0, a]$ satisfying Dirichlet and Neumann boundary conditions

$$
\left.\phi_{-}\right|_{x^{m}=0}=\left.0 \quad\left(\nabla_{m}+\frac{1}{2} V\right) \phi_{+}\right|_{x^{m}=0}=0 .
$$

Assertion of Lemma 4 follows immediately.

Under the conditions of Lemma $4 L_{a b}, \rho_{m m}$ and $f_{; m}$ vanish identically. We can calculate only the coefficients of $f \tau V^{2}, f V_{: a a} V, f V_{: a} V_{: a}$ and $f_{; m m} V^{2}$.

Corollary $c_{5,2}=\frac{1}{48}, c_{5,7}=\frac{1}{24}, c_{5,8}=\frac{5}{192}, c_{5,10}=\frac{1}{64}$

The rest of the universal constants can be calculated by using the conformal properties of the heat kernel which are exactly the same as for the usual boundary value problem [20].

Lemma 5. If $D(\epsilon)=e^{-2 f \epsilon} D$, then $\left.\frac{d}{d \epsilon}\right|_{\epsilon=0} a_{n}(1, D)=(m-n) a_{n}(f, D)$.

Under the conformal transformation the metric $g$ acquires a multiplier $e^{2 f \epsilon}$. $V$ is transformed to $e^{-f \epsilon} V$. Basic geometric quantities transform as [20]

$$
\begin{aligned}
\left(\left.\frac{d}{d \epsilon}\right|_{\epsilon=0} \Gamma\right)_{i j}^{k} & =\delta_{i k} f_{; j}+\delta_{j k} f_{; i}-\delta_{i j} f_{; k} \\
\left(\left.\frac{d}{d \epsilon}\right|_{\epsilon=0} L\right)_{a b} & =-\delta_{a b} f_{; m}-f L_{a b} \\
\left.\frac{d}{d \epsilon}\right|_{\epsilon=0}(E) & =-2 f E+\frac{1}{2}(m-2) f_{; i i} \\
\left.\frac{d}{d \epsilon}\right|_{\epsilon=0}(\tau) & =-2 f \tau+2(1-m) f_{; i i} \\
\left.\frac{d}{d \epsilon}\right|_{\epsilon=0}\left(\rho_{m m}\right) & =-2 f \rho_{m m}-f_{; a a}+(1-m) f_{; m m}
\end{aligned}
$$

where $\Gamma$ is the Christoffel connection. We need the following conformal relations

$$
\begin{aligned}
\left.\frac{d}{d \epsilon}\right|_{\epsilon=0} V^{2} \tau= & -4 f V^{2} \tau+2(1-m)\left[f_{; m m} V^{2}+2 f\left(V_{: a a} V+V_{: a} V_{: a}\right)\right. \\
& \left.-L_{: a a} f_{; m} V^{2}\right] \\
\left.\frac{d}{d \epsilon}\right|_{\epsilon=0} V^{2} \rho_{m m}= & -4 V^{2} \rho_{m m} f-2 f\left(V_{: a a} V+V_{: a} V_{: a}\right)+L_{a a} V f_{; m} \\
& +(1-m) V^{2} f_{; m m} \\
\left.\frac{d}{d \epsilon}\right|_{\epsilon=0} V^{2} E= & -4 V^{2} E f+(m-2) f\left(V_{: a a} V+V_{: a} V_{: a}\right) \\
& -\frac{1}{2}(m-2) L_{a a} f_{; m} V^{2}+\frac{1}{2}(m-2) f_{; m m} V^{2} \\
\left.\frac{d}{d \epsilon}\right|_{\epsilon=0} V^{2} L_{a a}^{2}= & -4 V^{2} L_{a a}^{2} f-2(m-1) V^{2} L_{a a} f_{; m}
\end{aligned}
$$




$$
\begin{aligned}
\left.\frac{d}{d \epsilon}\right|_{\epsilon=0} V^{2} L_{a b} L_{a b} & =-4 V^{2} L_{a b} L_{a b} f-2 V^{2} L_{a a} f_{; m} \\
\left.\frac{d}{d \epsilon}\right|_{\epsilon=0} V_{: a a} V & =-4 V_{: a a} V f-(m-3)\left(V_{: a a} V f+V_{: a} V_{: a} f\right) \\
\left.\frac{d}{d \epsilon}\right|_{\epsilon=0} V_{: a} V_{: a} & =-4 V_{: a} V_{: a} f+2\left(V_{: a a} V f+V_{: a} V_{: a} f\right) .
\end{aligned}
$$

To obtain the relation (49) we used integration by parts. Let $n=5$. By collecting the terms with $V_{: a a} V f$ and $V^{2} L_{: a a} f_{; m}$ we obtain

$$
\begin{aligned}
& 0=4(1-m) c_{5,2}-2 c_{5,3}+(m-2) c_{5,4}-(m-3) c_{5,7}+2 c_{5,8} \\
& 0=-2(1-m) c_{5,2}+c_{5,3}-\frac{1}{2}(m-2) c_{5,4}-2(m-1) c_{5,5}-2 c_{5,6}-(m-5) c_{5,9} .
\end{aligned}
$$

Solving these equation one obtains $c_{5,3}=1 / 192$ and $c_{5,9}=-5 / 384$. Below we collect for convenience of the reader all universal constants of Lemma 2

$$
\begin{aligned}
& c_{3,1}=\frac{1}{8}, c_{4,1}=\frac{1}{6}, c_{5,1}=\frac{1}{64}, c_{5,2}=\frac{1}{48}, c_{5,3}=\frac{1}{192}, c_{5,4}=\frac{1}{8}, \\
& c_{5,5}=-\frac{1}{256}, c_{5,6}=\frac{1}{128}, c_{5,7}=\frac{1}{24}, c_{5,8}=\frac{5}{192}, c_{5,9}=-\frac{5}{384}, c_{5,10}=\frac{1}{64} .
\end{aligned}
$$

\section{Conclusions}

In the present paper we have studied the heat kernel expansion for for an operator of Laplace type in the presence of semitransparent boundaries. We have determined the general form of the asymptotic expansion. Namely, we proved the validity of the asymptotic series (11). We have calculated explicitly several first terms of the expansion for the most general operator of Laplace type and arbitrary boundary potential. We believe that this is the most complete study done in this field so far.

Our methods of deriving the heat kernel coefficients admit extensive crosschecking. Most of the universal constants can be calculated by at least two independent methods. If needed, one can calculate the higher coefficients as well. As possible generalizations we can suggest the $\delta^{\prime}$ potentials or even general fourparameter family on matching conditions [1] on the hypersurface $\Sigma$. Another possible development of the present results can be the renormalization of quantum field theory in the presence of singular interactions [21]. We believe that semitransparent boundaries provide a more adequate framework for the renormalization than "abrupt" boundary conditions of Dirichlet or Neumann type. For most recent work on renormalization with singular potentials see [17].

\section{Acknowledgments}

We are grateful to Klaus Kirsten for fruitful discussions. One of the authors (D.V.) thanks the Alexander von Humboldt foundation and RFBR, grant 97-01- 
01186. He is also grateful to S. Randjbar-Daemi for kind hospitality at the Abdus Salam ICTP where this work was completed.

\section{Appendix A}

Some first non zero coefficients $X_{n, i}$ appearing in (32) read:

$$
\begin{gathered}
X_{1,1}=\frac{\alpha}{2}, X_{2,2}=\frac{-\alpha^{2}}{8}, X_{3,3}=\frac{\alpha}{16}+\frac{\alpha^{3}}{24}, X_{3,5}=\frac{-3 \alpha}{8}, X_{3,7}=\frac{5 \alpha}{16}, \\
X_{4,4}=\frac{-\alpha^{2}}{32}-\frac{\alpha^{4}}{64}, X_{4,6}=\frac{3 \alpha^{2}}{16}, X_{4,8}=\frac{-5 \alpha^{2}}{32}, \\
X_{5,5}=\frac{27 \alpha}{256}+\frac{\alpha^{3}}{64}+\frac{\alpha^{5}}{160}, X_{5,7}=\frac{-145 \alpha}{64}-\frac{3 \alpha^{3}}{32}, X_{5,9}=\frac{1085 \alpha}{128}+\frac{5 \alpha^{3}}{64}, \\
X_{5,11}=\frac{-693 \alpha}{64}, X_{5,13}=\frac{1155 \alpha}{256} .
\end{gathered}
$$

The Zeta functions defined in Eq. (34) are

$$
\begin{aligned}
\zeta(2, n) & =2 \zeta_{\mathrm{R}}(2 s+n) \\
\zeta(3, n) & =2 \zeta_{\mathrm{H}}\left(2 s+n-1 ; \frac{1}{2}\right) \\
\zeta(4, n) & =\zeta_{\mathrm{R}}(2 s+n-2) \\
\zeta(5, n) & =\frac{2}{3 !}\left(\zeta_{\mathrm{H}}\left(2 s+n-3 ; \frac{1}{2}\right)-\frac{1}{4} \zeta_{\mathrm{H}}\left(2 s+n-1 ; \frac{1}{2}\right)\right) \\
\zeta(6, n) & =\frac{2}{4 !}\left(\zeta_{\mathrm{R}}(2 s+n-4)-\zeta_{\mathrm{R}}(2 s+n-2)\right) \\
\zeta(7, n) & =\frac{2}{5 !}\left(\zeta_{\mathrm{H}}\left(2 s+n-5 ; \frac{1}{2}\right)-\frac{5}{2} \zeta_{\mathrm{H}}\left(2 s+n-3 ; \frac{1}{2}\right)+\frac{9}{16} \zeta_{\mathrm{H}}\left(2 s+n-1 ; \frac{1}{2}\right)\right),
\end{aligned}
$$

where $\zeta_{\mathrm{R}}$ and $\zeta_{\mathrm{H}}$ are the Riemann and Hurwitz zeta functions correspondingly.

\section{Appendix B}

In this appendix we give expressions for the heat kernel coefficients for the Dirichlet and Neumann boundary value problems. Let $M$ be a smooth compact Riemannian manifold with smooth boundary $\partial M$. Let $S$ be an endomorphism on $\mathcal{V}_{\partial M}$ and let $\phi_{; m}$ be a covariant derivative of $\phi$ with respect to inward unit nor-

mal. We define the modified Neumann boundary operator $\mathcal{B}^{+}$and the Dirichlet boundary operator $\mathcal{B}^{-}$by

$$
\mathcal{B}^{+} \phi:=\left.\left(\phi_{; m}+S \phi\right)\right|_{\partial M} \quad \mathcal{B}^{-} \phi:=\left.\phi\right|_{\partial M}
$$

We set $S=0$ for the Dirichlet boundary conditions to have a uniform notations. 
We need only the case of totally geodesic boundary $\left(L_{a b}=0\right)$. We drop certain boundary invariants which vanish under conditions of Lemma 4. Several first heat kernel coefficients are [20, 14, 22]

$$
\begin{aligned}
a_{0}\left(f, D, \mathcal{B}^{ \pm}\right)= & (4 \pi)^{-m / 2} \operatorname{Tr}(f)[M] \\
a_{1}\left(f, D, \mathcal{B}^{ \pm}\right)= & \pm \frac{1}{4}(4 \pi)^{-(m-1) / 2} \operatorname{Tr}(f)[\partial M] \\
a_{2}\left(f, D, \mathcal{B}^{ \pm}\right)= & (4 \pi)^{-m / 2} \frac{1}{6} \operatorname{Tr}\{(6 F E+F \tau)[M]+12 f S[\partial M]\} \\
a_{3}\left(f, D, \mathcal{B}^{ \pm}\right)= & \pm \frac{1}{384}(4 \pi)^{-(m-1) / 2} \operatorname{Tr}\left\{f\left(96 E+16 \tau+192 S^{2}\right)+24 F_{; m m}\right\}[\partial M] \\
a_{4}\left(f, D, \mathcal{B}^{ \pm}\right)= & (4 \pi)^{-m / 2} \frac{1}{360} \operatorname{Tr}\left(f \left(60 E_{; k k}+60 \tau E+180 E^{2}+30 \Omega^{2}+12 \tau_{; k k}\right.\right. \\
& \left.+5 \tau^{2}-2 \rho^{2}+2 R^{2}\right)[M]+\left(f \left(720 S E+120 S \tau+480 S^{3}\right.\right. \\
& \left.\left.\left.+120 S_{: a a}\right)+120 f_{; m m} S\right)[\partial M]\right) \\
a_{5}\left(f, D, \mathcal{B}^{ \pm}\right)= & \pm \frac{1}{5760}(4 \pi)^{-(m-1) / 2} \operatorname{Tr}\left\{f \left(360 E_{; m m}+1440 E_{; m} S+720 E^{2}\right.\right. \\
& +2880 E S^{2}+1440 S^{4}+240 E_{: a a}+240 \tau E+120 \Omega_{a b} \Omega_{a b} \\
& \left.+20 \tau^{2}-8 \rho^{2}+8 R^{2}+480 \tau S^{2}+960 S_{: a a} S+600 S_{: a} S_{: a}\right) \\
& \left.+f_{; m m}\left(360 E+360 S^{2}+60 \tau\right)+45 f_{; m m m m}\right\}[\partial M] .
\end{aligned}
$$

On manifold without a boundary one should keep volume contributions only.

\section{References}

[1] Albeverio S, Gesztesy F, Hoegh-Krohn R and Holden H 1988 Solvable Models in Quantum Mechanics (Springer, New York)

[2] Berezin F A and Faddeev L D 1961 Soviet Math. Dokl. 2372

[3] Vilenkin A and Shellard E P S 1994 Cosmic Strings and other Topological Defects (Cambridge University Press, Cambridge)

[4] Frolov V P and Fursaev D V 1998 Class. Quant. Grav. 152041

[5] Apps J S and Dowker J S 1998 Class. Quant. Grav. 151121

[6] Bordag M, Hennig D, Robaschik D 1992 J. Phys. A: Math. Gen. 25 44834498

[7] Jaekel M-T, Reynaud S 1992 Phys.Lett A 167 227-232

[8] Actor A A 1995 Phys.Rev. D52 3581-3590 
[9] Gilkey P B 1994 Invariance Theory, the Heat Equation and the Atiyah-Singer Index Theorem (CRC Press, Boca Raton)

[10] Scandurra M 1998 The ground state energy of a massive scalar field in the background of a semitransparent spherical shell hep-th/9811164, to appear in J.Phys.A

[11] Bordag M and Kirsten K 1998 The Ground state energy of a spinor field in the background of a finite radius flux tube hep-th/9812060, to appear in Phys.Rev.

[12] Schwinger J, DeRaad L L and Milton K A 1978 Ann. Phys. (N.Y.) 1151

[13] Bordag M, Kirsten K and Vassilevich D V 1999 Phys. Rev. D 59085011

[14] Branson T P, Gilkey P B and Vassilevich D V 1997 Boll. Unione Mat. Ital. (7) 11B Suppl. Fasc. 2 39, hep-th/9504029

[15] Grubb G 1986 Functional calculus of pseudo differential boundary problems (Progress in Math. 65, Birkhäuser)

[16] Albeverio S, Brzezniak Z and Dabrowski L 1994 J. Phys. A: Math. Gen. 27 4933

[17] Solodukhin S N 1999 Nucl. Phys B 539403

[18] Gaveau B and Schulman L S 1986 J. Phys. A: Math. Gen. 191833

[19] Bordag M, .Kirsten K and Elizalde E, 1996 J. Math. Phys. 37895

[20] Branson T P and Gilkey P B 1990 Commun. PDE 15245

[21] Symanzik K 1981 Nucl. Phys. B 1901

[22] Kirsten K 1998 Class. Quantum Grav. 15 L5. 